TRANSACTIONS OF THE

AMERICAN MATHEMATICAL SOCIETY

Volume 356, Number 8, Pages 3227-3242

S 0002-9947(03)03399-3

Article electronically published on December 12, 2003

\title{
EXISTENCE OF LOCAL SUFFICIENTLY SMOOTH SOLUTIONS TO THE COMPLEX MONGE-AMPÈRE EQUATION
}

\author{
SAOUSSEN KALLEL-JALLOULI
}

\begin{abstract}
We prove the $C^{\infty}$ local solvability of the $n$-dimensional complex Monge-Ampère equation $\operatorname{det}\left(u_{i \bar{j}}\right)=f(z, u, \nabla u), f \geq 0$, in a neighborhood of any point $z_{0}$ where $f\left(z_{0}\right)=0$.
\end{abstract}

In the present note we shall consider the complex Monge-Ampère equation

$$
\operatorname{det}\left(\frac{\partial^{2} \phi}{\partial z_{i} \partial \overline{z_{j}}}\right)=f(\operatorname{Re} z, \operatorname{Im} z, \phi, \nabla \phi)
$$

in an open set $\Omega$ of $\mathbb{C}^{n}, f$ a real-valued function, $z=\left(z_{1}, \ldots, z_{n}\right), z_{j}=x_{j}+y_{j}$, $\frac{\partial \phi}{\partial z_{j}}=\frac{1}{2}\left(\frac{\partial \phi}{\partial x_{j}}-i \frac{\partial \phi}{\partial y_{j}}\right) \quad$ and $\quad \frac{\partial \phi}{\partial \bar{z}_{j}}=\frac{1}{2}\left(\frac{\partial \phi}{\partial x_{j}}+i \frac{\partial \phi}{\partial y_{j}}\right)$.

Our main result is the following:

Theorem 1. Let $f \in C^{\infty}$ be nonnegative near a point $Z^{0}=\left(z_{0}, u_{0}, p_{0}\right) \in \mathbb{C}^{n} \times$ $\mathbb{R} \times \mathbb{R}^{2 n}$ and $f\left(Z^{0}\right)=0$. Then for any integer $s>n+3$, (0.1) has a plurisubharmonic (real-valued) solution $\phi \in H^{s}$ in a neighborhood of $z_{0}$, such that $\phi\left(z_{0}\right)=u_{0}$ and $\nabla \phi\left(z_{0}\right)=p_{0}($ seen as a function of $(\operatorname{Re} z, \operatorname{Im} z))$.

Theorem 2. Suppose in addition to the assumption in Theorem 1 that

$$
\partial_{(x, y)}^{\alpha} \partial_{u}^{l} \partial_{p}^{\beta} f\left(x_{0}, y_{0}, u_{0}, \nabla u_{0}\right)=0
$$

for all $|\alpha|+|\beta|+l \leq k-1$, and there exists $\alpha^{*} \in \mathbb{N}^{2 n}$ such that $\left|\alpha^{*}\right|=k$ and

$$
\partial_{(x, y)}^{\alpha^{*}} f\left(x_{0}, y_{0}, u_{0}, \nabla u_{0}\right) \neq 0 .
$$

Then (0.1) has a $C^{\infty}$ plurisubharmonic local real solution $u$ in a neighborhood of $\left(x_{0}, y_{0}\right)$.

Recall that in her paper 7] S. Kallel-Jallouli proved the local existence of a smooth plurisubharmonic solution to the problem (0.1) near a point $z_{0}$ in the particular case when $f(z, \phi, \nabla \phi)=K(z) g(z, \phi, \nabla \phi), g>0, K\left(z_{0}\right)=0$ and $d K\left(z_{0}\right) \neq 0$. The proof was essentially based on an inverse function theorem due to G. Nakumara and Y. Maeda [10].

Real Monge-Ampère equations of the form

$$
\operatorname{det}\left(\frac{\partial^{2} \phi}{\partial x_{i} \partial x_{j}}\right)=f(x, \phi, \nabla \phi)
$$

Received by the editors January 15, 2002 and, in revised form, February 5, 2003 and March 19, 2003.

2000 Mathematics Subject Classification. Primary 32W20.

(C)2003 American Mathematical Society 
were studied by many authors. When $f$ is nonnegative near a point in $\mathbb{R}^{n} \times \mathbb{R} \times \mathbb{R}^{n}$, C.S. Lin in [8] proved local existence of a sufficiently smooth solution to (0.2) in the two-dimensional case. The result was then extended in [5] by Hong and Zuily to spaces of arbitrary dimension. The method used is based on a Nash-Moser procedure (see [9]).

In this work, we shall use the same techniques. Since we derive $\phi$ with respect to two variables in each term of the matrix given in (0.1) and not only just one as in the real case $(0.2)$, some difficulties arise. The introduction of some adapted spaces and the use of some properties of the Laplace operator will enable us to overcome the difficulties.

We will start by giving several fundamental inequalities which play important roles in the proof of convergence of our iteration scheme.

\section{LINEAR THEORY}

We may assume that $Z^{0}=0$. Following C.S. Lin [8], by means of the change of unknown $\phi=\sum_{i=1}^{n-1} \sigma_{i}\left|z_{i}\right|^{2}+\varepsilon^{5} w$ and the change of variables $z_{i}=\varepsilon^{2} \tau_{i}, i=1, \ldots, n$, we can reduce $(0.1)$ to the following equation:

$$
\operatorname{det}\left(\phi_{i \bar{j}}\right)=\operatorname{det}\left(\left(1-\delta_{i}^{n}\right) \delta_{i}^{j} \sigma_{i}+\varepsilon w_{i \bar{j}}\right)=\tilde{f},
$$

where $\delta_{i}^{j}$ is the Kronecker symbol. The constants $\sigma_{i}$ are chosen such that

$$
\sigma_{1}>\sigma_{2}>\ldots>\sigma_{n-1}=1
$$

We shall consider

$$
G(w)=\frac{1}{\varepsilon} \operatorname{det}\left(\phi_{i \bar{j}}\right)-\frac{1}{\varepsilon} \tilde{f} \chi\left(x^{\prime}, y^{\prime}\right)
$$

in the neighborhood of the origin:

$$
\Omega=\left\{\left(x^{\prime}, x_{n}, y^{\prime}, y_{n}\right) \in \mathbb{R}^{2 n} ;\left|x_{i}^{\prime}\right| \leq \pi,\left|y_{i}^{\prime}\right| \leq \pi, x_{n}^{2}+y_{n}^{2} \leq r^{2}\right\},
$$

where $r$ will be chosen later and $\chi$ is a cut-off function vanishing near $x_{i}^{\prime}= \pm \pi$, $y_{i}^{\prime}= \pm \pi$ and equal to 1 near the origin.

Note that the projection of $\Omega$ on $\mathbb{R}_{\left(x_{n}, y_{n}\right)}^{2}$ is the disc

$$
D=\left\{\left(x_{n}, y_{n}\right) \in \mathbb{R}^{2} ; x_{n}^{2}+y_{n}^{2} \leq r^{2}\right\}
$$

and the boundary of $D$ is smooth and will help us to use some estimates related to the Laplace operator, established in [4] by Gilbarg and Trudinger.

The linearized operator of $G$ at $w$ is then

$$
L_{G}(w)=\sum_{i, j=1}^{n} \phi^{i j} \partial_{i} \partial_{j}+\sum_{i=1}^{n} a_{i} \partial_{\tau_{i}}+\sum_{i=1}^{n} b_{i} \partial_{\bar{\tau}_{i}}+c
$$

where $\left(\phi^{i j}\right)$ is the matrix of cofactors of $\left(\phi_{i \bar{j}}\right)$.

Now, for any smooth real-valued function $w$, the matrix $\left(\phi_{i \bar{j}}\right)$ is Hermitian and we can find a unitary matrix $T(\tau, \varepsilon)$ satisfying

$$
T(\tau, \varepsilon)\left(\phi_{i \bar{j}}\right)^{t} \bar{T}(\tau, \varepsilon)=\operatorname{diag}\left(\lambda_{1}, \ldots, \lambda_{n}\right) .
$$


Moreover, we have

Lemma 1.1. Suppose that $w$ is a smooth real-valued function with $|w|_{C^{3}} \leq 1$. Then $T(\tau, \varepsilon)$ is smooth in $(\tau, \varepsilon) \in \bar{\Omega} \times\left[0, \varepsilon_{0}\right]$ for some positive $\varepsilon_{0}$. Furthermore, with some constant independent of $w$ and $\varepsilon$ we have

$$
\begin{aligned}
& \left|T_{n n}(\tau, \varepsilon)-1\right|+\sum_{i, j=1}^{n}\left|\nabla_{x, y} T_{i j}(\tau, \varepsilon)\right|+\sum_{l=1}^{n-1}\left|\lambda_{l}(\tau, \varepsilon)-\sigma_{l}\right| \\
& +\left|\lambda_{n}(\tau, \varepsilon)\right|+\sum_{i=1}^{n-1}\left|T_{i n}(\tau, \varepsilon)\right| \leq c \varepsilon .
\end{aligned}
$$

Proof. Let $\Phi=\left(\phi_{i \bar{j}}\right)(\tau, \varepsilon)$. Since $\operatorname{det}(\Phi-\lambda I)(\tau, 0)=-\lambda \sum_{i=1}^{n-1}\left(\sigma_{i}-\lambda\right)$, by (1.2), the eigenvalues $\lambda_{i}(\tau, \varepsilon)$ are distinct and smooth for small $\varepsilon$ and the matrix $T(\tau, \varepsilon)$ is also smooth. It is also clear that $T_{i n}(\tau, 0)=0$ for $i=1, \ldots, n-1$ and $T_{n n}(\tau, 0)=1$, which implies (1.6).

Lemma 1.2. Let $|w|_{C^{2}} \leq 1$ and $\theta=\max _{\bar{\Omega}}|G(w)|$. Then the operator

$$
-L_{G}(w)-\theta \triangle
$$

where $\triangle=\sum_{i=1}^{n}\left(\frac{\partial^{2}}{\partial x_{i}^{2}}+\frac{\partial^{2}}{\partial y_{i}^{2}}\right)$, is a degenerate elliptic operator if $\varepsilon$ is small enough.

Proof. We have to prove

$$
A=\theta|\xi|^{2}+\sum_{i, j=1}^{n} \phi^{i j} \xi_{i} \overline{\xi_{j}} \geq 0 \quad \forall(\tau, \xi) \in \Omega \times \mathbb{C}^{n} .
$$

Let us set $\xi={ }^{t} T(\tau, \varepsilon) \widetilde{\xi}$ in (1.8) and let $\widetilde{\Phi}=\left(\phi^{i j}\right)$. We have $A=\theta|\xi|^{2}+$ ${ }^{t} \xi \widetilde{\Phi} \bar{\xi}=\theta|\xi|^{2}+{ }^{t} \widetilde{\xi} T \widetilde{\Phi}{ }^{t} \bar{T} \overline{\tilde{\xi}}$ but $\Phi \widetilde{\Phi}=\operatorname{det} \Phi . I d$, so by $(1.5), \operatorname{det} \Phi . I d=T \Phi^{t} \bar{T} T \widetilde{\Phi}^{t} \bar{T}=$ $\operatorname{diag}\left(\lambda_{i}\right) T \widetilde{\Phi}^{t} \bar{T}$, and

$$
\begin{aligned}
T \widetilde{\Phi}^{t} \bar{T} & =\operatorname{det} \Phi . \operatorname{diag}\left(\frac{1}{\lambda_{i}}\right)=\prod_{i=1}^{n} \lambda_{i} \cdot \operatorname{diag}\left(\frac{1}{\lambda_{i}}\right) \\
& =(\varepsilon G+\chi \widetilde{f}) \operatorname{diag}\left(\frac{1}{\lambda_{i}}\right) .
\end{aligned}
$$

Then,

$$
\begin{aligned}
A & =\theta|\widetilde{\xi}|^{2}+\operatorname{det} \Phi \cdot \sum_{i=1}^{n} \frac{\left|\widetilde{\xi}_{i}\right|^{2}}{\lambda_{i}} \\
& =\theta|\widetilde{\xi}|^{2}+\sum_{i=1}^{n-1} \operatorname{det} \Phi \frac{\left|\widetilde{\xi}_{i}\right|^{2}}{\lambda_{i}}+\prod_{i=1}^{n-1} \lambda_{i}\left|\widetilde{\xi}_{n}\right|^{2} \\
& =\left(\theta+\prod_{i=1}^{n-1} \lambda_{i}\right)\left|\widetilde{\xi}_{n}\right|^{2}+\sum_{i=1}^{n-1} \frac{\varepsilon G+\widetilde{f} \chi+\theta \lambda_{i}}{\lambda_{i}}\left|\widetilde{\xi}_{i}\right|^{2} .
\end{aligned}
$$

* If $\theta=0$, then $G(w)=0$ and $A \geq 0$ if $\widetilde{f} \geq 0$. 
* If $\theta>0$, by Lemma 1.1, if $\varepsilon$ is small enough, then

$$
\varepsilon G+\theta \lambda_{i} \geq \varepsilon G+\theta \sigma_{n-1}+O(\varepsilon) \geq \frac{1}{2} \theta>0,
$$

and $A \geq 0$.

(1.8) is consequently proved.

Now, we will study a boundary value problem for the degenerate elliptic operator $L_{G}(w)+\theta \triangle$. First we consider real-valued functions which are periodic in each variable $x_{i}^{\prime}, y_{i}^{\prime}, 1 \leq i \leq n-1$, with period $2 \pi$. Following [3], we introduce the space $\mathcal{H}_{s}(s \in \mathbb{N})$ which is the completion of the space of real-valued trigonometric polynomials $\sum_{l} \alpha_{l}\left(x_{n}, y_{n}\right) e^{i\left\langle l,\left(x^{\prime}, y^{\prime}\right)\right\rangle}$ with $\bar{\alpha}_{l}=\alpha_{-l} \in C_{0}^{\infty}(\bar{D}(0, r))$ with respect to the norm

$$
\left.\|u\|_{s}^{2}=\sum_{t+j \leq s} \sum_{l}\left(1+|l|^{2}\right)^{t}\left\|\alpha_{l}\right\|_{H^{j}}^{2} \stackrel{\circ}{D(0, r)}\right) .
$$

We can define $\stackrel{\circ}{\mathcal{H}}_{s}$ in the same way, taking $\alpha_{l}$ in $C_{0}^{\infty}(\stackrel{\circ}{D}(0, r))$. For $\rho$ in $\stackrel{\circ}{\mathcal{H}}_{1}$ we have of course $\rho\left(x^{\prime}, y^{\prime}, x_{n}, y_{n}\right)=0$ for $x_{n}^{2}+y_{n}^{2}=r^{2}$.

During this work, we will need two technical lemmas.

Lemma 1.3 (S. L. Sobolev [11]). If $u \in \mathcal{H}_{t}$ and $t \geq n+k+1$, then $u$ is of class $C^{k}$, and

$$
\max \left|\partial^{\alpha} u\right| \leq K_{\alpha, t}\|u\|_{t}, \text { for }|\alpha| \leq k .
$$

$K_{\alpha, t}$ is a constant independent of $u$.

Lemma 1.4 ([1], 6]). 1) If $u, v \in L^{\infty} \cap H^{t}(t>0)$, then $u v \in L^{\infty} \cap H^{t}$ and

$$
\|u v\|_{t} \leq K_{t}\left(\|u\|_{L^{\infty}}\|v\|_{t}+\|u\|_{t}\|v\|_{L^{\infty}}\right),
$$

where $K_{t}$ is a constant independent of $u, v$.

2) Let $H: \mathbb{R}^{m} \rightarrow \mathbb{C}$ be a $C^{\infty}$ function satisfying $H(0)=0$. If $\omega \in\left(L^{\infty} \cap H^{s}\right)^{m}$ $(s>0)$ and $\|\omega\|_{L^{\infty}} \leq M$, then

$$
\|H(\omega)\|_{s} \leq K(s, H, M)\|\omega\|_{s}
$$

where $K_{s}$ is a constant independent of $u$

Since each term of the diagonal of the matrix in (0.1) is the Laplace operator applied to $\frac{1}{4} \phi$, we shall need

Lemma 1.5 ([4]). Let $D$ be a bounded domain in $\mathbb{R}^{d}$ such that $\partial D \in C^{k+2}$ and $\rho \in H_{0}^{1}(D) \cap H^{k+2}(D)$. Then

$$
\|\rho\|_{H^{k+2}(D)} \leq C\left\{\|\rho\|_{L^{2}(D)}+\|\triangle \rho\|_{H^{k}(D)}\right\},
$$

where $C=C(d, k, \partial D)$.

The main result of this section is the following.

Theorem 1.6. Let $w$ be smooth, real-valued, periodic in $\left(x^{\prime}, y^{\prime}\right)$ and satisfy the inequality $\|w\|_{C^{n+4}} \leq 1$. Then for any $s_{0} \in \mathbb{N}$, one can find a constant $\varepsilon\left(s_{0}\right)$ such that given $g \in C^{\infty}(\bar{\Omega})$, the problem

$$
\left\{\begin{array}{l}
L_{G}(w) \rho+\theta \triangle \rho=g, \\
\rho \in \stackrel{\circ}{\mathcal{H}}_{1},
\end{array}\right.
$$


admits a unique solution $\rho \in \mathcal{H}_{s_{0}}$ provided that $0<\varepsilon<\varepsilon\left(s_{0}\right)$. Moreover, for $0<s<s_{0}$, the crucial inequality

$$
\|\rho\|_{s} \leq C_{s}\left\{\|g\|_{s}+\|(w)\|_{s+4}\|\rho\|_{L^{\infty}}\right\}
$$

holds for some constant $C_{s}$, independent of $w$ and $\varepsilon$.

Here $\|(w)\|_{s+4}$ is equal to zero if $s \leq n+1$ and to $\|w\|_{s+4}$ if $s>n+1$.

Later, in section 2, we will see how the inequality (1.10) is fundamental and makes the iteration scheme converge to a solution to our problem.

We will divide the proof of Theorem 1.6 into several lemmas. First of all, using the change of unknown function $\underline{\rho}=\rho e^{\lambda\left|\tau_{n}\right|^{2}}$ with $\tau_{n}=x_{n}+i y_{n}$, we reduce (1.9) to

$$
\begin{aligned}
& L(w) \underline{\rho}=\sum_{i, j=1}^{n}\left(\phi^{i j}+4 \delta_{i}^{j} \theta\right) \partial_{i} \partial_{\bar{j}} \underline{\rho}+\sum_{i=1}^{n} a_{i}^{\prime} \partial_{\tau_{i}} \underline{\rho} \\
& +\sum_{i=1}^{n} b_{i}^{\prime} \partial_{\bar{\tau}_{i}} \underline{\rho}+c^{\prime} \underline{\rho}=e^{\lambda\left|\tau_{n}\right|^{2}} g
\end{aligned}
$$

with

$$
\left\{\begin{array}{l}
a_{i}^{\prime}=a_{i}-\lambda \tau_{n}\left(\phi^{i n}+4 \delta_{i}^{n} \theta\right) \\
b_{i}^{\prime}=b_{i}-\lambda \overline{\tau_{n}}\left(\phi^{n i}+4 \delta_{i}^{n} \theta\right), \\
c^{\prime}=c+\left(\phi^{n n}+4 \theta\right)\left(\lambda^{2}\left|\tau_{n}\right|^{2}-\lambda\right)-\lambda \overline{\tau_{n}} a_{n}-2 \lambda b_{n} \tau_{n} .
\end{array}\right.
$$

Now, we replace $(1.9)$ by $\left(1.9^{\prime}\right)$ and write $\rho$ instead of $\underline{\rho}$. Instead of studying equation $\left(1.9^{\prime}\right)$ we will consider the following regularization of $\left(1.9^{\prime}\right)$.

Lemma 1.7. There exist three positive constants $r, \lambda, \varepsilon_{0}$ such that for $0<\varepsilon \leq \varepsilon_{0}$ and any real-valued $g$ in $C^{\infty}(\bar{\Omega})$ the regularized problem

$$
\left\{\begin{array}{l}
L_{\nu} \rho=L(w) \rho+\nu \triangle \rho=g \text { in } \Omega, \\
\rho \in \stackrel{\circ}{\mathcal{H}}_{1}(\Omega),
\end{array}\right.
$$

admits a unique (real-valued) solution $\rho \in C^{\infty}(\bar{\Omega})$ if $\nu>0$.

Proof. First of all, we recall that as an operator depending on $\partial_{x_{i}}$ and $\partial_{y_{i}}, i=$ $1, \ldots, n, L_{G}(w)$ is a real second order operator with real coefficients. Throughout the section $O(\varepsilon)$ means bounded by $K \varepsilon$, where $K$ is a constant independent of $\varepsilon, \lambda, r$. We shall take $\lambda r=1$. By (1.3), we can see that $\theta \leq M+O(\varepsilon)$, where $M$ is an absolute constant depending only on the function $f$. Moreover, by (1.1) we have $\phi^{i j}=O(\varepsilon)$ if $(i, j) \neq(n, n)$, together with its derivatives. We have also $\phi^{n n}=\Upsilon+O(\varepsilon)\left(\right.$ with $\left.\Upsilon=\prod_{i=1}^{n-1} \sigma_{i}\right), a_{j}=O(\varepsilon), b_{j}=O(\varepsilon), c_{j}=O(\varepsilon)$. Using (1.11), it follows that

$$
\left\{\begin{array}{l}
a_{i}^{\prime}=O(\varepsilon), 1 \leq i \leq n-1, \\
b_{i}^{\prime}=O(\varepsilon), 1 \leq i \leq n-1, \\
\left|a_{n}^{\prime}\right| \leq\left|a_{n}\right|+\lambda(4 \theta+\Upsilon)\left|\tau_{n}\right|, \text { so } a_{n}^{\prime}=O(1), \\
\left|b_{n}^{\prime}\right| \leq\left|b_{n}\right|+\lambda(4 \theta+\Upsilon)\left|\tau_{n}\right|, \text { so } b_{n}^{\prime}=O(1),
\end{array}\right.
$$

and $c^{\prime}=\left(\phi^{n n}+4 \theta\right)\left(\lambda^{2}\left|\tau_{n}\right|^{2}-\lambda\right)+O(\varepsilon)$, so $-\operatorname{Re} c^{\prime} \geq \lambda(\Upsilon+4 \theta)\left(1-\lambda\left|\tau_{n}\right|^{2}\right)+$ $O(\varepsilon)$. 
Now, since $\rho$ is $2 \pi$-periodic in each variable $x_{i}^{\prime}, y_{i}^{\prime}, i=1, \ldots, n-1$, and vanishes when $x_{n}^{2}+y_{n}^{2}=r^{2}$, integration by parts then gives

$$
\begin{aligned}
& \left(-L_{\nu} \rho, \rho\right)_{0}=\int_{\Omega} \nu|\nabla \rho|^{2} d x d y \\
& +\int_{\Omega} \underbrace{\sum_{i, j=1}^{n}\left(\phi^{i j}+4 \delta_{i}^{j} \theta\right) \partial_{i} \rho \partial_{\bar{j}} \rho d x d y}_{(1)} \\
& -\underbrace{\int_{\Omega} \sum_{i=1}^{n} a_{i}^{\prime} \partial_{i} \rho . \rho d x d y}_{(2)}-\underbrace{\int_{\Omega} \sum_{i=1}^{n} b_{i}^{\prime} \partial_{\bar{i}} \rho \cdot \rho d x d y}_{(2)^{\prime}} \\
& -\underbrace{\int_{\Omega}\left(c^{\prime}+\frac{1}{2} \sum_{i, j=1}^{n} \partial_{\bar{j}} \partial_{i} \phi^{i j}\right) \rho^{2} d x d y}_{(3)} .
\end{aligned}
$$

Let us set $\nabla_{\tau} \rho={ }^{t} T \widetilde{\rho}$, where $\nabla_{\tau}=\left(\begin{array}{c}\partial_{\tau_{1}} \\ \vdots \\ \partial_{\tau_{n}}\end{array}\right)$. We get, following the proof of Lemma 1.2,

$$
\begin{aligned}
(1) & =\int_{\Omega}{ }^{t}\left(\nabla_{\tau} \rho\right)(\widetilde{\Phi}+4 \theta I d) \overline{\nabla_{\tau} \rho} d x d y \\
& =\int_{\Omega}{ }^{t} \widetilde{\rho} T(\widetilde{\Phi}+4 \theta I d)\left({ }^{t} \bar{T}\right) \overline{\tilde{\rho}} d x d y \\
& =\int_{\Omega}\left\{\left(4 \theta+\prod_{i=1}^{n-1} \lambda_{i}\right)\left|\widetilde{\rho}_{n}\right|^{2}+\sum_{i=1}^{n-1} \frac{\varepsilon G+4 \theta \lambda_{i}+\chi \widetilde{f}}{\lambda_{i}}\left|\widetilde{\rho}_{i}\right|^{2} d x d y\right\} .
\end{aligned}
$$

Now, an integration by parts gives

$$
\begin{aligned}
(2) & =\frac{1}{2} \sum_{i=1}^{n-1} \int_{\Omega} \partial_{i} a_{i}^{\prime} \rho^{2} d x d y-\underbrace{\int_{\Omega} a_{n}^{\prime} \partial_{n} \rho . \rho d x d y}_{(4)}, \\
\partial_{n} \rho & =\sum_{i=1}^{n} T_{i n} \widetilde{\rho}_{i}=T_{n n} \widetilde{\rho}_{n}+\sum_{i=1}^{n-1} \sum_{j=1}^{n} T_{i n} \bar{T}_{j i} \partial_{\tau_{j}} \rho \\
& =T_{n n} \widetilde{\rho}_{n}+\sum_{i, j=1}^{n-1} T_{i n} \bar{T}_{j i} \partial_{\tau_{j}} \rho+\sum_{i=1}^{n-1} T_{i n} \bar{T}_{n i} \partial_{\tau_{n}} \rho .
\end{aligned}
$$

Since ${ }^{t} T \bar{T}=I d$, then $\sum_{j=1}^{n} T_{i n} \bar{T}_{n i}=1$ and

$$
\partial_{n} \rho=\frac{1}{\left|T_{n n}\right|^{2}}\left\{T_{n n} \widetilde{\rho}_{n}+\sum_{i, j=1}^{n-1} T_{i n} \bar{T}_{j i} \partial_{j} \rho\right\} .
$$


Now,

$$
(4)=-\underbrace{\int_{\Omega} a_{n}^{\prime} \rho \frac{T_{n n}}{\left|T_{n n}\right|^{2}} \widetilde{\rho}_{n} d x d y}_{(5)}-\underbrace{\int_{\Omega} a_{n}^{\prime} \rho \sum_{i, j=1}^{n-1} \frac{T_{i n} \bar{T}_{j i}}{\left|T_{n n}\right|^{2}} \partial_{\tau_{j}} \rho d x d y}_{(6)} .
$$

We have

$$
\begin{aligned}
(6) & =\frac{1}{2} \int_{\Omega} \sum_{i, j=1}^{n-1} a_{n}^{\prime} \frac{T_{i n} \bar{T}_{j i}}{\left|T_{n n}\right|^{2}} \partial_{\tau_{j}} \rho^{2} d x d y \\
& =-\frac{1}{2} \sum_{i, j=1}^{n-1} \int_{\Omega} \partial_{j}\left(a_{n}^{\prime} \frac{T_{i n} \bar{T}_{j i}}{\left|T_{n n}\right|^{2}}\right) \rho^{2} d x d y,
\end{aligned}
$$

which clearly implies, using Lemma 1.1, that

$$
|(6)| \leq C \varepsilon \int_{\Omega} \rho^{2} d x d y
$$

For (5) we shall use the inequality $a . b \leq \alpha a^{2}+\frac{1}{\alpha} b^{2}$ with $\alpha=\frac{1}{2} \prod_{i=1}^{n-1} \lambda_{i}>0$ and the estimates $a_{n}^{\prime}=O(1), T_{n n}=1+O(\varepsilon)$. We get

$$
\begin{aligned}
|(5)| & \leq \int_{\Omega} \frac{\left|a_{n}^{\prime} \rho\right|}{\left|T_{n n}\right|}\left|\widetilde{\rho}_{n}\right| d x d y \\
& \leq \frac{1}{2} \int_{\Omega} \prod_{i=1}^{n-1} \lambda_{i}\left|\widetilde{\rho}_{n}\right|^{2} d x d y+\int_{\Omega} \frac{2}{\prod_{i=1}^{n-1} \lambda_{i}} \frac{a_{n}^{\prime 2}}{\left|T_{n n}\right|} \rho^{2} d x d y \\
& \leq \frac{1}{2} \int_{\Omega} \prod_{i=1}^{n-1} \lambda_{i}\left|\widetilde{\rho}_{n}\right|^{2} d x d y+C \int_{\Omega} \rho^{2} d x d y .
\end{aligned}
$$

$(2)^{\prime}$ is estimated similarly to (2). Let us now look at (3). By the discussion at the beginning of the proof we get

$$
\operatorname{Re}(3) \geq\left[\lambda(\Upsilon+4 \theta)-(\Upsilon+4 \theta) \lambda^{2}\left|\tau_{n}\right|^{2}+O(\varepsilon)\right] \int_{\Omega} \rho^{2} d x d y .
$$

Summing up, we conclude that

$$
\left(-L_{\nu} \rho, \rho\right) \geq \int_{\Omega}\left\{\nu|\nabla \rho|^{2}+\frac{1}{2} \prod_{i=1}^{n-1} \lambda_{i}\left|\widetilde{\rho}_{n}\right|^{2}+[(\Upsilon+4 \theta) \lambda-C+O(\varepsilon)] \rho^{2} d x d y\right\} d x d y .
$$

We take $\lambda$ big enough to have coercitivity, and then we apply the Lax-Milgram theorem to get a unique solution $\rho$ in $\stackrel{\circ}{\mathcal{H}}_{1}(\Omega)$ to the problem $\left(1.9^{\prime \prime}\right)$. Provided $g$ is smooth, the regularity of $\rho$ follows from the theory of elliptic equations.

Proof of Theorem 1.6. Suppose $\left(1.10_{s}\right)$ is valid for the regularized problem $\left(1.9^{\prime \prime}\right)$ with a uniform constant $C_{s}$ for $\left.\left.\nu \in\right] 0,1\right]$. Then by letting $\nu$ go to zero we shall get a solution of the original problem which of course will satisfy $\left(1.10_{s}\right)$.

For the regularized problem we shall use induction on $s$. When $s=0,(1.10)_{0}$ follows from (1.13). Actually we have the strong estimate

$$
\int_{\Omega}\left|\widetilde{\rho}_{n}\right|^{2} d x d y+\|\rho\|_{L^{2}}^{2} \leq C_{0}\|g\|_{0}^{2}
$$


Denote by $C_{s}$ (resp. $C$ ) any constant which is independent of $\nu$ and $\varepsilon$; it will change from line to the next. Assume that $\left(1.10_{s}^{\prime}\right)$

$$
\sum_{\substack{|\alpha| \leq s-1 \\ \alpha_{n}=\alpha_{2 n}=0}} \int_{\Omega}\left|\left(\widetilde{\partial^{\alpha} \rho}\right)_{n}\right|^{2} d x d y+\|\rho\|_{s-1}^{2} \leq C_{s-1}\left\{\|g\|_{s-1}^{2}+\|(w)\|_{s+3}^{2}\|\rho\|_{L^{\infty}}^{2}\right\}
$$

for all $\left.\varepsilon \in] 0, \varepsilon\left(s_{0}\right)\right]$ and $0 \leq s-1 \leq s_{0}$ with $s \geq 1$. We shall find a new $\varepsilon\left(s_{0}\right)$ which makes $\left(1.10_{s}^{\prime}\right)$ true.

Denote by $\rho^{s}$ any derivative of order $\alpha$ with $|\alpha|=s$ and $\alpha_{n}=\alpha_{2 n}=0$. Note that $\partial^{\alpha}=\partial_{x_{1}}^{\alpha_{1}} \ldots \partial_{x_{n-1}}^{\alpha_{n-1}} . \partial_{y_{1}}^{\alpha_{n+1}} \ldots \partial_{y_{n-1}}^{\alpha_{2 n-1}}$

Using (1.13), we get

$$
\left|\left(-L_{\nu} \rho^{s}, \rho^{s}\right)\right| \geq C \int_{\Omega}\left[\left(\rho^{s}\right)^{2}+\frac{1}{2} \prod_{i=1}^{n-1} \lambda_{i}\left(\widetilde{\partial^{\alpha} \rho}\right)_{n}^{2}\right] d x d y .
$$

On the other hand,

$$
\left(-L_{\nu} \rho^{s}, \rho^{s}\right)=-\left(g^{s}, \rho^{s}\right)-\left(\left[L_{\nu}, \partial^{\alpha}\right] \rho, \rho^{s}\right) .
$$

Let us look at the second term of (1.15). We have

$$
\left[L_{\nu}, \partial^{\alpha}\right]=\sum_{\substack{\beta \leq \alpha \\|\beta| \geq 1}} C_{\alpha \beta}\left[\partial^{\beta}\left(\phi^{i j}\right) \partial_{i} \partial_{\bar{j}}+\partial^{\beta} a_{j}^{\prime} \partial_{j}+\partial^{\beta} b_{j}^{\prime} \partial_{\bar{j}}+\partial^{\beta} c\right] \partial^{\alpha-\beta} .
$$

For $|\beta|=1$, we have, using integrations by parts,

$$
\begin{gathered}
H=\left(\partial^{\beta}\left(\phi^{i j}\right) \partial_{i} \partial_{\bar{j}} \rho^{s-1}, \rho^{s}\right)=\left(\partial\left(\phi^{i j}\right) \partial_{i} \partial_{\bar{j}} \rho^{s-1}, \partial \rho^{s-1}\right) \\
=-\underbrace{\int_{\Omega} \partial_{\bar{j}} \rho^{s-1} \partial_{i} \partial\left(\phi^{i j}\right) \partial \rho^{s-1} d x d y}_{(7)}-\int_{\Omega} \partial_{\bar{j}} \rho^{s-1} \partial\left(\phi^{i j}\right) \partial_{i} \partial \rho^{s-1} d x d y \\
=-(7)+\underbrace{\int_{\Omega} \rho^{s-1} \partial_{\bar{j}} \partial\left(\phi^{i j}\right) \partial_{i} \partial \rho^{s-1} d x d y}_{(8)}+\int_{\Omega} \rho^{s-1} \partial\left(\phi^{i j}\right) \partial \partial_{i} \partial_{\bar{j}} \rho^{s-1} d x d y \\
=-(7)+(8)-\underbrace{\int_{\Omega} \partial_{i} \partial_{\bar{j}} \rho^{s-1} \partial^{2}\left(\phi^{i j}\right) \rho^{s-1} d x d y}_{(9)}-H .
\end{gathered}
$$

Then,

$$
\begin{gathered}
2 H=(8)-(7)-(9), \\
(9)=-\int_{\Omega} \partial_{\bar{j}} \rho^{s-1} \partial_{i} \partial^{2}\left(\phi^{i j}\right) \rho^{s-1} d x d y-\int_{\Omega} \partial_{\bar{j}} \rho^{s-1} \partial^{2}\left(\phi^{i j}\right) \partial_{i} \rho^{s-1} d x d y,
\end{gathered}
$$

which can be estimated by $C \varepsilon\|\rho\|_{s}^{2}$. We estimate the terms (7), (8) in the same way to obtain

$$
|H| \leq C \varepsilon\|\rho\|_{s}^{2} .
$$

For $2 \leq|\beta| \leq n+1$, since $\|w\|_{C^{n+3}} \leq 1$ and $|\alpha|-|\beta|+2 \leq s$, we have

$$
\left|\left(\partial^{\beta}\left(\phi^{i j}\right) \partial_{i} \partial_{\bar{j}} \rho^{s-|\beta|}, \rho^{s}\right)\right| \leq C \varepsilon\|\rho\|_{s}^{2} .
$$


If $|\beta|>n+1$, we can write

$$
A=\left|\left(\partial^{\beta}\left(\phi^{i j}\right) \partial_{i} \partial_{\bar{j}} \rho^{s-|\beta|}, \rho^{s}\right)\right| \leq\|\rho\|_{s}\left\|\partial^{\beta}\left(\phi^{i j}\right) \partial_{i} \partial_{\bar{j}} \rho^{s-|\beta|}\right\|_{L^{2}} .
$$

Using Lemma 1.4 with $u=\partial^{2}\left(\phi^{i j}\right)$ and $v=\rho$, we get

$$
A \leq C\left\|\rho^{s}\right\|_{L^{2}}\left(\varepsilon\|w\|_{C^{4}}\|\rho\|_{s}+\|\rho\|_{L^{\infty}}\|w\|_{s+4}\right) .
$$

The lower order terms in $L_{\nu}$ are estimated in a similar way. Summing up, we conclude, using (1.15) to (1.17), that

$$
\left|\left(-L_{\nu} \rho^{s}, \rho^{s}\right)\right| \leq C\left\{\varepsilon\|\rho\|_{s}^{2}+\left\|\rho^{s}\right\|_{L^{2}}\left[\left\|g^{s}\right\|_{L^{2}}+\|(w)\|_{s+4}\|\rho\|_{L^{\infty}}\right]\right\} .
$$

It remains to estimate the derivatives $\partial_{x_{n}}^{k_{1}} \partial_{y_{n}}^{k_{2}} \rho^{s-k_{1}-k_{2}}$, for $k_{1}+k_{2}=1, \ldots, s$. From (1.12) we get

$$
\partial_{n} \rho^{s-1}=\frac{1}{\left|T_{n n}\right|^{2}}\left\{T_{n n}\left(\widetilde{\rho^{s-1}}\right)_{n}+\sum_{i, j=1}^{n-1} T_{i n} \bar{T}_{j i} \partial_{j} \rho^{s-1}\right\},
$$

but since $\rho$ is real-valued, then

$$
\partial_{x_{n}} \rho^{s-1}=\operatorname{Re}\left(\partial_{\tau_{n}} \rho^{s-1}\right) \quad \text { and } \quad \partial_{y_{n}} \rho^{s-1}=-\operatorname{Im}\left(\partial_{\tau_{n}} \rho^{s-1}\right),
$$

$\mathrm{SO}$

$$
\left\{\begin{array}{l}
\left\|\partial_{x_{n}} \rho^{s-1}\right\|_{L^{2}} \leq C\left(\left\|\left(\widetilde{\rho^{s-1}}\right)_{n}\right\|_{L^{2}}+\varepsilon\|\rho\|_{s}\right), \\
\left\|\partial_{y_{n}} \rho^{s-1}\right\|_{L^{2}} \leq C\left(\left\|\left(\widetilde{\rho^{s-1}}\right)_{n}\right\|_{L^{2}}+\varepsilon\|\rho\|_{s}\right) .
\end{array}\right.
$$

Using the induction estimate $\left(1.10_{s-1}^{\prime}\right)$, we get, for $k_{1}+k_{2}=1$,

$$
\left\|\partial_{x_{n}}^{k_{1}} \partial_{y_{n}}^{k_{2}} \rho^{s-1}\right\|_{L^{2}} \leq C\left\{\|g\|_{s-1}+\|(w)\|_{s+3}\|\rho\|_{L^{\infty}}+\varepsilon\|\rho\|_{s}\right\} .
$$

Denote $\partial_{n}^{k}=\partial_{x_{n}}^{k_{1}} \partial_{y_{n}}^{k_{2}}$ for $k=k_{1}+k_{2}$ and write the original équation $\left(1.9^{\prime \prime}\right)$ in another way:

$$
\begin{aligned}
\triangle_{n} \rho= & \frac{4}{\phi^{n n}+4 \theta+4 \nu}\left\{g-\sum_{i<n}\left(\frac{1}{4} \phi^{i i}+\theta+\nu\right) \triangle_{i} \rho-\sum_{i \neq j} \phi^{i j} \partial_{i} \partial_{\bar{j}}\right. \\
& \left.-a_{j}^{\prime} \partial_{\tau_{j}} \rho-b_{j}^{\prime} \partial_{\overline{\tau_{j}}} \rho-c^{\prime} \rho\right\}=F,
\end{aligned}
$$

with $\triangle_{i}=\partial^{2} / \partial x_{i}^{2}+\partial^{2} / \partial y_{i}^{2}, \quad i=1, \ldots, n$.

Applying $\partial^{s-1}$ to (1.20), we obtain using Lemma 1.5, with $d=2$ and $D=$ $\left\{\left(x_{n}, y_{n}\right) ; x_{n}^{2}+y_{n}^{2} \leq r^{2}\right\}$,

$$
\left\|\partial_{n}^{k+2} \rho^{s-k}\right\|_{L^{2}}^{2} \leq C\left\{\left\|\rho^{s-k}\right\|_{L^{2}}^{2}+\sum_{0 \leq l \leq k}\left\|\partial_{n}^{l} \partial^{s-k} F\right\|_{L^{2}}^{2}\right\} .
$$

Now, we are able to prove (1.19) for $k=2, \ldots, s$ by induction on $k$, using (1.21), $\left(1.10_{s-1}^{\prime}\right)$ and Lemma 1.4 (1.25). We get

$$
\sum_{k=2}^{s}\left\|\partial_{n}^{k} \rho^{s-1}\right\|_{L^{2}} \leq C_{s}\left\{\|g\|_{s}+\|(w)\|_{s+3}\|\rho\|_{L^{\infty}}+\varepsilon\|\rho\|_{s}\right\} .
$$

Using (1.14), (1.18), (1.19) and (1.22), we get $\left(1.10_{s}^{\prime}\right)$ for $0 \leq s \leq s_{0}$ and $0 \leq$ $\varepsilon \leq \varepsilon\left(s_{0}\right)$ if $\varepsilon\left(s_{0}\right)$ is small enough. 


\section{ExistenCE OF SUFFICIENTLY SMOOTH SOLUTIONS}

In this section we shall construct, using the results of section 1, a sequence which converges to a solution to our problem.

Let $E$ be the space of all smooth functions in $\bar{\Omega}$ which are periodic in $\left(x^{\prime}, y^{\prime}\right)$, and let $\tau$ and $\sigma>1$ be two constants which will be chosen later. One can define a family of smoothing operators $S_{n}$ from $E$ to $E$ such that with $\mu_{n}=\sigma^{\tau^{n}}$ and for $0 \leq s_{1}, s_{2} \leq s_{0}$

$$
\begin{gathered}
\left\|S_{n} u\right\|_{s_{1}} \leq C_{s_{2}}\|u\|_{s_{2}}, \text { if } s_{1} \leq s_{2}, \\
\left\|S_{n} u\right\|_{s_{1}} \leq C_{s_{1}} \mu_{n}^{s_{1}-s_{2}}\|u\|_{s_{2}}, \text { if } s_{1} \geq s_{2}, \\
\left\|S_{n} u-u\right\|_{s_{1}} \leq C_{s_{2}} \mu_{n}^{s_{1}-s_{2}}\|u\|_{s_{2}}, \quad \text { if } s_{1} \leq s_{2},
\end{gathered}
$$

where the constant $C_{s_{i}}$ is independent of $u, n, \sigma, \tau$. We will construct $w_{n}, n=$ $0,1, \ldots$, by induction on $n$ as follows. Starting with $w_{0}=0$, suppose $w_{0}, w_{1}, \ldots, w_{n}$ have been chosen and define $w_{n+1}$ as follows:

$$
w_{n+1}=w_{n}+S_{n} \rho_{n}
$$

where $\rho_{n}$ is the solution of

$$
L\left(w_{n}\right) \rho_{n}=L_{G}\left(w_{n}\right) \rho_{n}+\theta_{n} \triangle \rho_{n}=g_{n} \text { in } \Omega
$$

satisfying

$$
\rho_{n} \in \stackrel{\circ}{\mathcal{H}}_{1}
$$

given by Theorem 1.6, when

$$
g_{n}=-G\left(w_{n}\right), \theta_{n}=\sup _{\bar{\Omega}}\left|G\left(w_{n}\right)\right| .
$$

In order to ensure that the $w_{k}$ are well defined, there are several things to be verified. We prove first the following result.

Lemma 2.1. Suppose $\left\|w_{k}\right\|_{C^{n+3}} \leq 1$ for $k=0,1, \ldots, m$. Then for all $k=0,1, \ldots, m$ we have

$$
\begin{gathered}
\left\|g_{k}\right\|_{s} \leq C_{s}\left\{\left\|g_{0}\right\|_{s}+\left\|w_{k}\right\|_{s+2}\right\}, \\
\left\|w_{k+1}\right\|_{s+4} \leq C_{s}^{k+1} \mu_{k+1}^{\beta}\left\|g_{0}\right\|_{s} \text { for some } \beta>\frac{4}{\tau-1}, \\
\left\|g_{k+1}\right\|_{L^{2}} \leq \mu_{k+1}^{-\kappa}\left\|g_{0}\right\|_{s^{*}} \text { for some positive constants } \kappa \text { and } s^{*} .
\end{gathered}
$$

Proof. a) $\left\|g_{k}\right\|_{s} \leq\left\|G\left(w_{0}\right)\right\|_{s}+\left\|G\left(w_{k}\right)-G\left(w_{0}\right)\right\|_{s}$. Using the Taylor formula for $G$, Lemma 1.4 and the hypothesis $w_{0}=0$, we easily get (2.8).

b) Using (2.2) and (2.4), we get

$$
\left\|w_{k+1}\right\|_{s+4} \leq C_{s}\left(\left\|w_{k}\right\|_{s+4}+\left\|S_{k} \rho_{k}\right\|_{s+4}\right) \leq C_{s}\left(\left\|w_{k}\right\|_{s+4}+\mu_{k}^{4}\left\|\rho_{k}\right\|_{s}\right) .
$$

$*$ If $s \leq n+1,\left(1.10_{s}\right)$ gives

$$
\left\|\rho_{k}\right\|_{s} \leq C_{s}\left\|g_{k}\right\|_{s} \leq C^{\prime}\left\{\left\|g_{0}\right\|_{s}+\left\|w_{k}\right\|_{s+2}\right\},
$$

so, since $\mu_{k}>1$, then

$$
\left\|w_{k+1}\right\|_{s+4} \leq C_{s} \mu_{k}^{4}\left(\left\|g_{0}\right\|_{s}+\left\|w_{k}\right\|_{s+4}\right) .
$$

$*$ If $s>n+1$, we have by Lemma 1.3,

$$
\left\|\rho_{k}\right\|_{L^{\infty}} \leq C\left\|\rho_{k}\right\|_{n+1},
$$


so $\left(1.10_{s}\right)$ gives $\left\|\rho_{k}\right\|_{s} \leq C\left(\left\|g_{k}\right\|_{s}+\left\|w_{k}\right\|_{s+4}\right)$, and using (2.8) we get the same estimate (2.11). Iteration of this estimate yields, since $w_{0}=0$,

$$
\left\|w_{k+1}\right\|_{s+4} \leq C_{s}^{k}(k+1) \mu_{0}^{4} \ldots \mu_{k}^{4}\left\|g_{0}\right\|_{s} \leq C_{s}^{k}(k+1) \sigma^{4 \frac{\tau^{k+1}}{\tau-1}}\left\|g_{0}\right\|_{s},
$$

which proves $(2.9)$.

c) In view of (2.5) and (2.7) we have

$$
-g_{k+1}=G\left(w_{k}\right)+L_{G}\left(w_{k}\right) S_{k} \rho_{k}+Q\left(w_{k}, S_{k} \rho_{k}\right),
$$

where $Q$ is the quadratic error, and

$$
-g_{k+1}=G\left(w_{k}\right)+L\left(w_{k}\right) \rho_{k}+L\left(w_{k}\right)\left(S_{k}-I\right) \rho_{k}-\theta_{k} \triangle S_{k} \rho_{k}+Q\left(w_{k}, S_{k} \rho_{k}\right) .
$$

The sum of the first two terms in the right-hand side vanishes, so

$$
\left\|g_{k+1}\right\|_{0} \leq \underbrace{\left\|L\left(w_{k}\right)\left(S_{k}-I\right) \rho_{k}\right\|_{0}}_{(1)}+\underbrace{\theta_{k}\left\|\triangle S_{k} \rho_{k}\right\|_{0}}_{(2)}+\underbrace{\left\|Q\left(w_{k}, S_{k} \rho_{k}\right)\right\|_{0}}_{(3)} .
$$

Since $\left\|w_{k}\right\|_{n+1} \leq 1$, then

$$
\begin{gathered}
|(1)| \leq C\left\|\left(S_{k}-I\right) \rho_{k}\right\|_{2} \leq C_{s^{*}} \mu_{k}^{-\left(s^{*}-2\right)}\left\|\rho_{k}\right\|_{s^{*}}, \\
|(2)| \leq \theta_{k}\left\|S_{k} \rho_{k}\right\|_{2} \leq \theta_{k} \mu_{k}^{2}\left\|\rho_{k}\right\|_{0} \\
|(3)| \leq C\left\|S_{k} \rho_{k}\right\|_{L^{\infty}}\left\|S_{k} \rho_{k}\right\|_{4} .
\end{gathered}
$$

It follows from $\left(1.10_{s^{*}}\right)$, with $s^{*}$ to be determined, that

$$
\left\|g_{k+1}\right\|_{0} \leq C_{s^{*}}\left\{\mu_{k}^{-\left(s^{*}-2\right)}\left(\left\|g_{k}\right\|_{s^{*}}+\left\|w_{k}\right\|_{s^{*}+4}\right)+\theta_{k} \mu_{k}^{2}\left\|g_{k}\right\|_{0}+\mu_{k}^{n+5}\left\|g_{k}\right\|_{0}^{2}\right\} .
$$

Now by (2.8) and (2.9)

$$
\left\|g_{k}\right\|_{s^{*}}+\left\|w_{k}\right\|_{s^{*}+4} \leq C\left(\left\|g_{0}\right\|_{s^{*}}+\left\|w_{k}\right\|_{s^{*}+4}\right) \leq C C_{s^{*}}^{k} \mu_{k}^{\beta}\left\|g_{0}\right\|_{s^{*}},
$$

so

$$
\left\|g_{k+1}\right\|_{0} \leq C_{s^{*}}\left\{\mu_{k}^{-\left(s^{*}-2-\beta\right)} C^{k}\left\|g_{0}\right\|_{s^{*}}+\theta_{k} \mu_{k}^{2}\left\|g_{k}\right\|_{0}+\mu_{k}^{n+5}\left\|g_{k}\right\|_{0}^{2}\right\} .
$$

Now by (2.7), and using Lemma 1.3, we get

$$
\theta_{k+1}=\left\|g_{k+1}\right\|_{L^{\infty}} \leq\left\|g_{k+1}\right\|_{n+1},
$$

so

$$
\theta_{k+1} \leq\left\|L\left(w_{k}\right)\left(S_{k}-I\right) \rho_{k}\right\|_{n+1}+\theta_{k}\left\|\triangle S_{k} \rho_{k}\right\|_{n+1}+\left\|Q\left(w_{k}, S_{k} \rho_{k}\right)\right\|_{n+1} .
$$

Using the same estimates us before, we get

$$
\theta_{k+1} \leq C_{s^{*}}\left\{\mu_{k}^{-\left(s^{*}-n-3-\beta\right)} C_{s^{*}}^{k}\left\|g_{0}\right\|_{s^{*}}+\theta_{k} \mu_{k}^{n+3}\left\|g_{k}\right\|_{0}+\mu_{k}^{6+2 n}\left\|g_{k}\right\|_{0}^{2}\right\} .
$$

Let us choose $\kappa$ and $s^{*}$ such that

$$
\left\{\begin{array}{l}
(2-\tau) \kappa-8-2 n>0, \\
s^{*}-4-n-\beta-\kappa \tau>0,
\end{array}\right.
$$

and set

$$
d_{k+1}=\max \left(\mu_{k+1}^{\kappa}\left\|g_{k+1}\right\|_{0}, \mu_{k+1}^{\kappa} \theta_{k+1}\right) .
$$

Noting that $\mu_{k+1}=\mu_{k}^{\tau}$, we have by $(2.12)$

$\mu_{k+1}^{\kappa}\left\|g_{k+1}\right\|_{0} \leq C_{s^{*}}\left\{\mu_{k}^{\kappa \tau-\left(s^{*}-2-\beta\right)} C^{k}\left\|g_{0}\right\|_{s^{*}}+\theta_{k} \mu_{k}^{\kappa \tau+2}\left\|g_{k}\right\|_{0}+\mu_{k}^{\kappa \tau+n+5}\left\|g_{k}\right\|_{0}^{2}\right\}$. 
By (2.14) we get

$$
\left\|g_{k+1}\right\|_{0} \leq C_{s^{*}}\left\{C^{k+1} \mu_{k}^{-2-n}\left\|g_{0}\right\|_{s^{*}}+\theta_{k} \mu_{k}^{2 \kappa-2 n-4}\left\|g_{k}\right\|_{0}+\theta_{k} \mu_{k}^{2 \kappa-n-1}\left\|g_{k}\right\|_{0}^{2}\right\} .
$$

Taking $\sigma$ large enough, it follows that

$$
\mu_{k+1}^{\kappa}\left\|g_{k+1}\right\|_{0} \leq \frac{1}{4}\left\|g_{0}\right\|_{s^{*}}+d_{k}^{2}
$$

In the same way, using (2.13) and (2.14) we get

$$
\mu_{k+1}^{\kappa} \theta_{k+1} \leq \frac{1}{4}\left\|g_{0}\right\|_{s^{*}}+d_{k}^{2}
$$

and then we have proved that

$$
d_{k+1} \leq \frac{1}{4}\left\|g_{0}\right\|_{s^{*}}+d_{k}^{2}
$$

Now

$$
g_{0}=-G\left(w_{0}\right)=\frac{1}{\varepsilon} \breve{f}\left(\varepsilon^{2}(x, y) ; \varepsilon^{4} \sum_{i \leq n-1} \sigma_{i}\left(x_{i}^{2}+y_{i}^{2}\right) ; 2 \varepsilon^{2} \sum_{i \leq n-1} \sigma_{i}\left(x_{i}, y_{i}\right)\right)
$$

and $\breve{f}(0 ; 0 ; 0)=0$, so we can suppose that $\left\|g_{0}\right\|_{s^{*}} \leq 1$. Moreover, we assume that

$$
\max \left(\mu_{0}^{2 \kappa} \theta_{0}, \mu_{0}^{2 \kappa}\left\|g_{0}\right\|\right) \leq \frac{1}{4 \max \left(1, K_{0, n+1}\right)} .
$$

Here $K_{0, n+1}$ is given by Lemma 1.3. By (2.15) and (2.17) we can conclude that $d_{1} \leq \frac{1}{2}\left\|g_{0}\right\|_{s^{*}}$. By induction it is easy to see that we have

$$
d_{k+1} \leq \frac{1}{2}\left\|g_{0}\right\|_{s^{*}},
$$

which proves (2.10).

Proof of Theorem 1. We shall prove by induction that for some constant $\Gamma$

$$
\left\|w_{k}\right\|_{2 n+4} \leq \Gamma \text {. }
$$

Since $w_{0}=0$, we may suppose that $\left(2.20_{k}\right)$ is true for all $k=0,1, \ldots, m$. By (2.4), and using the Gagliardo-Nirenberg inequality, we get

$$
\left\|w_{m+1}\right\|_{s^{\prime}} \leq \sum_{k=0}^{m}\left\|S_{k} \rho_{k}\right\|_{s^{\prime}} \leq C \sum_{k=0}^{m}\left\|\rho_{k}\right\|_{s^{\prime}} \leq C \sum_{k=0}^{m}\left\|\rho_{k}\right\|_{s^{*}}^{\frac{s^{\prime}}{s^{*}}}\left\|\rho_{k}\right\|_{0}^{1-\frac{s^{\prime}}{s^{*}}} .
$$

By $\left(1.10_{s^{*}}\right),(2.8)$, and $(2.9)$

$$
\left\|\rho_{k}\right\|_{s^{*}} \leq C\left(\left\|g_{0}\right\|_{s^{*}}+\left\|w_{k}\right\|_{s^{*}+4}\right) \leq C^{\prime}\left\|g_{0}\right\|_{s^{*}}\left(1+C^{k+1} \mu_{k}^{\beta}\right) \leq C_{1}^{k} \mu_{k}^{\beta}\left\|g_{0}\right\|_{s^{*}},
$$

and by (2.10), $\left\|\rho_{k}\right\|_{0} \leq C\left\|g_{k}\right\|_{0} \leq C^{\prime} \mu_{k}^{-\kappa}\left\|g_{0}\right\|_{s^{*}}$. It follows that

$$
\left\|w_{m+1}\right\|_{s^{\prime}} \leq C \sum_{k=0}^{m} C_{1}^{k \frac{s^{\prime}}{s^{*}}} \mu_{k}^{\beta \frac{s^{\prime}}{s^{*}}-\kappa\left(1-\frac{s^{\prime}}{s^{*}}\right)}\left\|g_{0}\right\|_{s^{*}} .
$$

We may fix the constants now. We first choose $\tau=\frac{4}{3}, \beta=12+\delta$, with $\delta>0$ small, $\kappa=12+3 n+\delta$; then the first inequality in (2.14) is satisfied, and for the second one we have to take $s^{*}>5 n+33$. 
Let us go back to (2.21); then, taking $\frac{s^{\prime}}{s^{*}}$ sufficiently small and $\sigma$ large, the series is convergent and we get $\left\|w_{m+1}\right\|_{s^{\prime}} \leq C_{s^{*}}\left\|g_{0}\right\|_{s^{*}}$. The induction step is completed if we take $\varepsilon\left(s^{*}\right)$ so small that $C_{s^{*}}\left\|g_{0}\right\|_{s^{*}} \leq \Gamma$ (see (2.18)).

We have $w_{m} \rightarrow w$ in $H_{s}(\Omega)$ and

$$
\|w\|_{s} \leq C_{s^{*}}\left\|g_{0}\right\|_{s^{*}} .
$$

By (2.10), $g_{m} \rightarrow 0$; then, $w$ is a solution of $G(w)=0$ if $s \geq n+3$, and so $\phi=\sum_{i=0}^{n-1} \sigma_{i}\left|z_{i}\right|^{2}+\varepsilon^{5} w\left(\varepsilon^{-2} z\right)$ is a solution of the original Monge-Ampère equation, which is plurisubharmonic if $\varepsilon$ is small enough since $\varepsilon\left|\frac{\partial^{2} w}{\partial z_{i} \partial z_{j}}\right|=O(\varepsilon)$. This completes the proof of Theorem 1.

\section{Existence of a $C^{\infty}$ LOCAl solution}

We shall use the result of C.J. Xu and C. Zuily [12, [13, which we recall briefly. Let us consider a nonlinear partial differential equation

$$
F\left(x, y, u, \nabla u, D^{2} u\right)=0,
$$

where $F$ is $C^{\infty}$. To any solution $u$ we can associate the vector fields

$$
X_{j}=\sum_{k} \frac{\partial F}{\partial u_{j k}} \partial_{k} .
$$

Then we get

Theorem $3.1([12])$. Suppose $u \in C_{l o c}^{\rho}(\Omega)$ with $\rho>\operatorname{Max}(4, r+2)$ for some constant $r \geq 0$, and that the brackets of the $X_{j}$, up to the order $r$, span the tangent space at each point of $\Omega$. Then $u$ belongs to $C^{\infty}(\Omega)$.

We can replace $\frac{\partial^{2} \phi}{\partial z_{i} \partial \overline{z_{j}}}$ by the sum

$$
\frac{1}{4}\left\{\frac{\partial^{2} \phi}{\partial x_{i} \partial x_{j}}+\frac{\partial^{2} \phi}{\partial y_{i} \partial y_{j}}+i \frac{\partial^{2} \phi}{\partial x_{i} \partial y_{j}}\right\}
$$

so, by noting that $y_{i}=x_{i+n}, 1 \leq i \leq n$, we obtain for our particular equation

$$
\begin{gathered}
X_{i}=\phi^{i i} \frac{\partial}{\partial x_{i}}+\sum_{\substack{j=1 \\
j \neq i}}^{n} \frac{\phi^{i j}+\overline{\phi^{i j}}}{2} \frac{\partial}{\partial x_{j}}+\sum_{\substack{j=1 \\
j \neq i}}^{n} \frac{i \phi^{i j}-i \overline{\phi^{i j}}}{2} \frac{\partial}{\partial x_{j+n}}, \\
X_{i+n}=\phi^{i i} \frac{\partial}{\partial x_{i+n}}+\sum_{\substack{j=1 \\
j \neq i}}^{n} \frac{\phi^{i j}+\overline{\phi^{i j}}}{2} \frac{\partial}{\partial x_{j+n}}+\sum_{\substack{j=1 \\
j \neq i}}^{n} \frac{i \phi^{i j}-i \overline{\phi^{i j}}}{2} \frac{\partial}{\partial x_{j}} .
\end{gathered}
$$

Hence

$$
X_{i}=\varepsilon w_{n \bar{n}} A_{i} \partial_{x_{i}}+\varepsilon \sum_{l=1}^{2 n} \sum_{j \neq i} w_{i \bar{j}} P_{i j l}\left(D^{2} w\right) \partial x_{l} \quad \text { for } i \notin\{n, 2 n\},
$$


and

$$
\left\{\begin{array}{l}
X_{n}=A_{n} \partial_{x_{n}}+\varepsilon \sum_{\substack{j \neq \delta \\
l}} w_{\delta \bar{j}} P_{\delta j l}\left(D^{2} w\right) \partial x_{l}, \\
X_{2 n}=A_{n} \partial_{x_{2 n}}+\varepsilon \sum_{\substack{j \neq \delta \\
l}} w_{\delta \bar{j}} P_{\delta j l}^{\prime}\left(D^{2} w\right) \partial x_{l} .
\end{array}\right.
$$

Here $P_{\delta j l}, P_{\delta j l}^{\prime}$ are polynomials in $D^{2} w, A_{n}=\operatorname{det}\left(\delta_{i}^{j} \sigma_{i}+\varepsilon w_{i \bar{j}} ; 1 \leq i, j \leq n-1\right)$ and $A_{l}$ is the cofactor of $\sigma_{l}+\varepsilon w_{l \bar{l}}$ in $A_{n}$. In order to compute the Lie algebra generated by the $X_{i}$, we need some lemmas.

Lemma 3.2. Let $\partial_{(x, y)}^{\alpha} \partial_{u}^{l} \partial_{p}^{\beta} f(0,0,0)=0$ for all $|\alpha|+|\beta|+l \leq k-1$. Assume $w \in H^{s}$ with $s \geq n+k+1$. Then

$$
\left|\partial^{\alpha} w\right| \leq C_{\alpha} \varepsilon^{2 k-1}, \quad \text { for all }|\alpha| \leq k
$$

Proof. Using (2.18), it follows from the hypothesis that the Taylor expansion of $g_{0}$ is on the form

$$
\begin{aligned}
& g_{0}=\varepsilon^{4 k-1} \sum_{|\alpha|+|\beta|+l=k} C_{l \alpha \beta} \varepsilon^{2 l}(x, y)^{\alpha}\left(x^{\prime}, y^{\prime}\right)^{\beta}\left|x^{\prime}+i y^{\prime}\right|^{2 l} \int_{0}^{1}(1-\lambda)^{k} \\
& \times \partial_{(x, y)}^{\alpha} \partial_{u}^{l} \partial_{p}^{\beta} f\left(\lambda \varepsilon^{2}(x, y) ; 2 \lambda \varepsilon^{4} \sum_{i<n} \sigma_{i}\left(x_{i}^{2}+y_{i}^{2}\right) ; 2 \lambda \varepsilon^{2} \sigma_{i}\left(x^{\prime}, y^{\prime}\right)\right) d \lambda,
\end{aligned}
$$

and (3.3) follows from the inequality (2.22) and Lemma 1.3.

Lemma 3.3. In addition to the assumption in Lemma 3.1, let

$$
\partial_{y_{n}}^{k} f(0,0,0)>0 \text {. }
$$

Then, if $\varepsilon$ is small enough,

$$
\partial_{y_{n}}^{k} w_{n \bar{n}} \geq C \varepsilon^{2 k-1}
$$

with a constant $C$ independent of $\varepsilon$.

Proof. Since $A_{n}=\Upsilon+\varepsilon \sum_{i, j} w_{i \bar{j}} A_{i j}\left(D^{2} w\right)$, we rewrite the equation $\operatorname{det}\left(\phi_{i \bar{j}}\right)=f$ as

$$
w_{n \bar{n}}+\varepsilon \sum_{i, j, k, m} w_{i \bar{j}} w_{k \bar{m}} A_{i j k m}\left(D^{2} w\right)=\frac{f}{\varepsilon \Upsilon} .
$$

Now

$$
\begin{aligned}
& \frac{1}{\varepsilon} f\left(\varepsilon^{2} x ; \varepsilon^{4} \sigma_{i} x_{i}^{2}+\varepsilon^{5} w,\left(2 \varepsilon^{2} \sigma_{i} x_{i}+\varepsilon^{3} \partial_{x_{i}} w\right)_{i \neq n, 2 n}, \varepsilon^{3} \partial_{x_{n}} w, \varepsilon^{3} \partial_{x_{2 n}} w\right) \\
& =g_{0}+\varepsilon^{4} w \Phi_{1}(x, \varepsilon, w, \nabla w)+\varepsilon^{2} \sum_{p=2}^{2 n+1} w_{p-1} \Phi_{p}(x, \varepsilon, w, \nabla w), \Phi_{j} \in C^{\infty} .
\end{aligned}
$$

Applying to both sides of (3.6), (3.7) the operator $\partial_{y_{n}}^{k}$ and using (3.3), we get

so,

$$
\partial_{y_{n}}^{k} w_{n \bar{n}} \geq \frac{1}{\Upsilon} \partial_{y_{n}}^{k} g_{0}-C_{k}\left(\varepsilon^{2 k+3}+\varepsilon^{2 k+1}+\varepsilon^{4 k-1}\right) ;
$$

$$
\partial_{y_{n}}^{k} w_{n \bar{n}} \geq \frac{1}{\Upsilon} \varepsilon^{2 k-1} \partial_{y_{n}}^{k} f(0,0,0)-C_{k}\left(\varepsilon^{2 k+3}+\varepsilon^{2 k+1}+\varepsilon^{4 k-1}\right) .
$$

But $f(0,0,0)=0$. Then $k \geq 1$, and $(3.5)$ is proved. 
Proof of Theorem 2. Without loss of generality we can assume that $\alpha^{*}=(0, \ldots, 0, k)$ and $\partial_{y_{n}}^{k} f(0,0,0)>0$. Choose $s$ so big that $s \geq k+n+4$. By means of Theorem 1 we can get a solution $w \in H^{s}$. Moreover, $w$ satisfies (3.3) and (3.5).

* When $n>2$ the polynomials $B_{i j l}$ occurring in (3.1) and (3.2) are at least of degree 1 . Thus, for each $i \in\{1, \ldots, n-1, n+2, \ldots, 2 n-1\}$, using induction on the size of the bracket, one can prove that

$$
\left(a d X_{2 n}\right)^{k}\left(X_{i}\right)=\varepsilon \sigma_{i} \partial_{y_{n}}^{k} \omega_{n \bar{n}} A_{n}^{k} \partial_{x_{i}}+\varepsilon \sum_{i=1}^{2 n} \sum_{\substack{|\alpha|+|\beta| \leq k \\ 1 \leq a, p, b, q \leq 2 n}} C_{\alpha \beta a b p q}^{i} \partial^{\alpha} \omega_{a \bar{b}} \partial^{\beta} \omega_{p \bar{q}} \partial_{x_{i}},
$$

where the $C_{\alpha \beta a b p q}^{i}$ are still polynomials in $D^{\gamma} \omega ;|\gamma| \leq k$.

From (3.3) and (3.5) the sum in the right hand side is $O\left(\varepsilon^{4 k-1}\right)$ while the coefficient of the first term is $\geq C_{0} \varepsilon^{2 k}$. It follows that the vector fields

$$
\left[\left(a d X_{2 n}\right)^{k}\left(X_{i}\right)\right]_{i=1, \ldots, n-1, n+1, \ldots, 2 n-1}, X_{n} \text { and } X_{2 n}
$$

span all the tangent space. Theorem 2 follows then from Theorem 3.1 and Lemma 1.3 .

* If $n=2$, a direct computation of the determinant of the vector fields

$$
\left[\left(a d X_{4}\right)^{k}\left(X_{i}\right)\right]_{i=1,3}, X_{2} \text { and } X_{4}
$$

shows that it is equal to

$$
\varepsilon^{2} \sigma^{2 k+2}\left(\partial_{y_{n}}^{k} \omega_{n \bar{n}}\right)^{2}+\varepsilon^{3} \sum_{\substack{|\alpha|+|\beta|+|\gamma| \leq k \\ 1 \leq a, p, b, q, i, j \leq 2 n}} C_{\alpha \beta a b p q} \partial^{\alpha} \omega_{a \bar{b}} \partial^{\beta} \omega_{p \bar{q}} \partial^{\gamma} \omega_{i \bar{j}},
$$

which by (3.3) and (3.5) doesn't vanish, and we conclude in the same way.

* If $n=1$, we don't need the hypothesis of Theorem 2 to conclude.

\section{Supplement}

As noted by Bedford and Taylor in [2], if we set $\left|z_{i}\right|=e^{x_{i}}$ and $\psi\left(x_{1}, \ldots, x_{n}\right)=$ $\phi\left(e^{x_{1}}, \ldots, e^{x_{n}}\right)$, then it is easily checked that

$$
\frac{\partial^{2} \psi}{\partial x_{i} \partial x_{j}}=4 z_{i} \overline{z_{j}} \frac{\partial^{2} \phi}{\partial z_{i} \partial \overline{z_{j}}}
$$

and

$$
\operatorname{det}\left(\frac{\partial^{2} \psi}{\partial x_{i} \partial x_{j}}\right)=4^{n} e^{2 x_{1}} \ldots e^{2 x_{n}} \operatorname{det}\left(\frac{\partial^{2} \phi}{\partial z_{i} \partial \overline{z_{j}}}\right)
$$

so, we can state, as a consequence of Theorem 1.2,

Theorem 3 ([5], Theorem 1). Let $f \in C^{\infty}$ be nonnegative near a point $Z^{0}=$ $\left(y_{0}, u_{0}, p_{0}\right) \in \mathbb{R}^{n} \times \mathbb{R} \times \mathbb{R}^{n}$ and $f\left(Z^{0}\right)=0$. Then for any integer $s>n+3$, the problem

$$
\operatorname{det}\left(\frac{\partial^{2} \psi}{\partial y_{i} \partial y_{j}}\right)=f(y, u, \nabla u)
$$

has a convex solution $u \in H^{s}$ in a neighborhood of $y_{0}$, such that $u\left(y_{0}\right)=u_{0}$ and $\nabla u\left(y_{0}\right)=p_{0}$. 
Theorem 4 ([5], Theorem 2). Suppose in addition to the assumption in Theorem 3 that $\partial_{y}^{\alpha} \partial_{u}^{l} \partial_{p}^{\beta} f\left(y_{0}, u_{0}, \nabla u_{0}\right)=0$ for all $|\alpha|+|\beta|+l \leq k-1$ and there exists $\alpha^{*} \in \mathbb{N}^{n}$ such that $\left|\alpha^{*}\right|=k$ and $\partial_{y}^{\alpha^{*}} f\left(y_{0}, u_{0}, \nabla u_{0}\right) \neq 0$. Then (4.1) has a $C^{\infty}$ convex local solution $u$ in a neighborhood of $y_{0}$.

Finally, I wish to express my thanks to the referee for some helpful comments.

\section{REFERENCES}

[1] S. Alinhac, P. Gérard: Opérateurs pseudo-différentiels et théorème de Nash-Moser, Inter Editions et Editions du CNRS,1991.

[2] E. Bedford and B.A. Taylor: The Dirichlet problem for a complex Monge-Ampère equation, Inventiones Mathematicae, 37, 1-44 1979. MR 56:3351

[3] L. Bers, F. John, M. Schechter: Partial differential equations, Lectures in Applied Mathematics, vol 3, Amer. Math. Soc., 1957. MR 29:346

[4] D. Gilbarg, N.S. Trudinger: Elliptic partial differential equations of second order, second edition, Springer Verlag Berlin, Heidelberg, New York 1983. MR 86c:35035

[5] J. Hong and C.Zuily: Existence of $C^{\infty}$ local solutions for the Monge-Ampère equation, Invent. Math. 89, 645-661, 1987. MR 88j:35056

[6] L. Hörmander: On the Nash-Moser implicit function theorem, Annales Academia Scientiarum.Fennicae, Serie A I, Math. 10, 67-97, 1984.

[7] S. Kallel-Jallouli: Existence of $C^{\infty}$ local solutions of the complex Monge-Ampère equation, Proc. Amer. Math. Soc., 131, 1103-1108, 2003.

[8] C.S. Lin: The local isometric embedding in $\mathbb{R}^{3}$ of 2-dimensional Riemannian manifolds with nonnegative curvature, J. Differential Geometry, 21, 213-230, 1985. MR 87m:53073

[9] J. Moser: A new technique for the construction of solutions of nonlinear partial differential equations, Proc. Natl. Acad. Sc. USA 47, 1842-1831, 1961. MR 24:A2695

[10] G. Nakamura, Y. Maeda: Local smooth isometric embedding of low dimensional Riemannian manifolds into euclidean spaces, Transactions of the A.M.S., 313, 1-51, 1989. MR 90f:58171

[11] S.L. Sobolev: On a theorem of functional analysis, Mat. Sb. (N.S.) 4, 471-497, 1938. (Russian); English transl., Amer. Math. Soc. Transl (2) 34, 39-68, 1963.

[12] C.J. Xu: Régularité des solutions pour les équations aux dérivées partielles quasi linéaires non elliptiques du second ordre, C. R. Acad. Sci. Paris Sér. 1 Math. 300, 267-270, 1985.

[13] C.J. Xu, C. Zuily: Smoothness up to the boundary for solutions of the nonlinear and nonelliptic Dirichlet problem, Trans. of the Amer. Math. Soc. 308, 243-257, 1988. MR 90c:35034

Faculté des Sciences, Campus Universitaire, 1060 Tunis, Tunisie

E-mail address: Saoussen.Kallel@fst.rnu.tn 